\title{
Cyclopolymerization of a bisacrylate through selective formation of a 19-membered ring
}

\author{
Bungo Ochiai, Tetsuya Shiomi and Hasumi Yoshita
}

Radical polymerization of a bisacrylate monomer trans-1,2-bisacryloyloxyethylcarbamoyloxycyclohexane proceeded via cyclopolymerization. The repeating unit consisted of a 19-membered ring structure. Notably, highly selective cyclopolymerization proceeded at a monomer concentration of $0.033 \mathrm{moll}^{-1}$ and produced the corresponding polymer in high yield. The selectivity was validated by size-exclusion chromatography, and nuclear magnetic resonance and matrix-assisted laser desorption ionization time-of-flight mass spectroscopic analyses.

Polymer Journal (2016) 48, 859-862; doi:10.1038/pj.2016.45; published online 18 May 2016

\section{INTRODUCTION}

Cyclopolymerization facilitates preparation of polymers bearing cyclic structures, which serve as rigid skeletons for thermal and mechanical stability, templates for reactions and selective capturing of ions and molecules. ${ }^{1-4}$ For supramolecular applications, macrocycles larger than 12-membered rings are typically necessary, as indicated by the supramolecular chemistry of various low-molecular-weight hosts, such as crown ethers ${ }^{4-6}$ and cyclodextrins. ${ }^{7,8}$ Cyclopolymerization requires very high selectivity of cyclization to avoid crosslinking; hence, cyclopolymerization-producing macrocycles cannot be performed without specific design of monomers and/or polymerization methods. The simplest approach is polymerization under highly diluted conditions, in which, for example, various polymers bearing crown ether cavities have been prepared with bifunctional monomers bearing polyether spacers. However, the low concentration often limits the yields of polymers. ${ }^{4,9}$ Effective strategies for improvement of the cyclization tendency involve conformational orientation of two polymerization groups with cyclic structures ${ }^{10-21}$ or templates such as metal cations ${ }^{22,23}$ and selective alternating copolymerization. ${ }^{24,25}$ In the most common method, namely, the design of oriented monomers, additional constraints by steric hindrance ${ }^{12,13,19}$ and hydrogen bonding ${ }^{17}$ are effective in improving the cyclization tendency.

Among these methodologies, we focus on the design of oriented bifunctional monomers accessible via simple one-step reactions suitable for practical applications. ${ }^{15,17,20}$ A bismethacrylate monomer, BMCH (trans-1,2-bismethacryloyloxyethylcarbamoyloxycyclohexane), can be prepared via the reaction of 2-methacryloyloxyethylisocyanate and trans-1,2-cyclohexanediol. The radical polymerization of $\mathrm{BMCH}$ proceeded through the selective cyclopolymerization because of the orientation of the methacrylate moieties by the cyclohexane ring and the hydrogen bonding of the urethane groups and gave a polymer consisting of repeating 19-membered ring units. ${ }^{17}$ To expand the applicability of this design, we prepared BACH (trans-1,2-bisacryloyloxyethylcarbamoyloxycyclohexane) as an acrylate analog of $\mathrm{BMCH}$ and explored its cyclopolymerization, giving a polymer composed of repeating units bearing a 19 -membered ring.

\section{EXPERIMENTAL PROCEDURE}

Materials

2,2'-Azobisisobutyronitrile (AIBN) was recrystallized from methanol. 1,4-Dioxane was distilled over sodium under a nitrogen atmosphere. Dehydrated $\mathrm{N}, \mathrm{N}$-dimethyl formamide was stored under a nitrogen atmosphere. 2-Acryloyloxyethylisocyanate was supplied by Showa Denko (Tokyo, Japan). Other reagents were used as received.

\section{Measurements}

${ }^{1} \mathrm{H}(400 \mathrm{MHz})$ and ${ }^{13} \mathrm{C}(100 \mathrm{MHz})$ nuclear magnetic resonance (NMR) spectra were measured on a JEOL (Tokyo, Japan) ECX-400 instrument using tetramethylsilane as an internal standard at ambient temperature in $\mathrm{CDCl}_{3}$. Fourier-transform infrared spectra were measured on a Horiba (Kyoto, Japan) FT-210 instrument. Elemental analysis was performed on a Perkin-Elmer (Waltham, MA, USA) CHNS/O2400II analyzer. Size-exclusion chromatography measurements were performed on a Tosoh (Tokyo, Japan) DP-8020 pump system equipped with a Viscotek (Houston, TX, USA) TDA model 300 detector and polystyrene-gel tandem columns (Tosoh TSK gels GMHXL, G4000XL, G3000XL and G2500XL) using tetrahydrofuran (THF) as an eluent at $40^{\circ} \mathrm{C}$. Polystyrene standards were used for calibration. Matrix-assisted laser desorption/ionization time-of-flight (MALDI-TOF) mass spectra were measured on a Bruker Daltonics (Billerica, MA, USA) ultrafleXtreme spectrometer using the linear mode. The acceleration voltage was $25 \mathrm{kV}$. The mass was calibrated with angiotensin II (1047.189 Da), angiotensin I (1297.486 Da), substance P (1348.642), bombesin (1620.860 Da), ACTH clip(1-17) (2097.427 Da), somatostatin-28 (3149.574 Da) and insulin (5734.508 Da). The sample solution was prepared by mixing the polymer solution in THF $\left(10 \mathrm{mg} \mathrm{ml}^{-1}\right)$, dithranol in $\operatorname{THF}\left(10 \mathrm{mg} \mathrm{ml}^{-1}\right)$ and $\mathrm{CF}_{3} \mathrm{COONa}$ in $\operatorname{THF}\left(1 \mathrm{mg} \mathrm{ml}^{-1}\right)$ in a $1 / 10 / 1$ 
volume ratio. A $1 \mu \mathrm{l}$ aliquot of the solution was dropped on a measurement plate and was dried at room temperature in the dark.

\section{Synthesis of BACH}

To a 100-ml two-necked flask equipped with a dropping funnel and a threeway cock, trans-1,2-cyclohexanediol $(2.32 \mathrm{~g}, 20.0 \mathrm{mmol})$ was added, and the flask was filled with nitrogen. Next, dichloromethane $(40 \mathrm{ml})$ and dibutyltin dilaurate $(11.8 \mu \mathrm{l}, 20 \mu \mathrm{mol})$ were added. A dichloromethane $(4.0 \mathrm{ml})$ solution of 2-acryloyloxyethylisocyanate $(5.93 \mathrm{~g}, 42.0 \mathrm{mmol}$ ) was added dropwise from the dropping funnel, and the mixture was stirred at $30^{\circ} \mathrm{C}$ for $24 \mathrm{~h}$ under a nitrogen atmosphere. After the reaction, diethyl ether and brine were added, and the organic layer was dried over $\mathrm{MgSO}_{4}$. Volatile substances were evaporated, and the residue was subjected to silica gel column chromatography eluted with chloroform. Crude $\mathrm{BACH}$ was purified via recrystallization from a mixed solvent of diethyl ether and hexane to obtain white crystals of $\mathrm{BACH}$ (3.78 g, $9.48 \mathrm{mmol}, 47.4 \%)$.

Melting point $=67.5-68.0{ }^{\circ} \mathrm{C}$. ${ }^{1} \mathrm{H}$ NMR ( $\delta$ in p.p.m.): $1.20-1.48$ and $1.62-1.80 \quad\left(4 \mathrm{H}, \quad\right.$ br, $\left.\quad-\mathrm{CH}_{2}\left(\mathrm{CH}_{2}\right)_{2} \mathrm{CH}_{2}-\right), \quad 1.20-1.48$ and $2.00-2.18$ $\left(4 \mathrm{H}, \quad b r,-\mathrm{CH}_{2}\left(\mathrm{CH}_{2}\right)_{2} \mathrm{CH}_{2}-\right), \quad 3.34-3.56\left(4 \mathrm{H}, \quad\right.$ br, $\left.-\mathrm{COOCH}_{2} \mathrm{CH}_{2} \mathrm{NH}-\right)$, $4.10-4.30 \quad\left(4 \mathrm{H}, \quad \mathrm{t}, \quad J=5.5 \mathrm{~Hz}, \quad-\mathrm{COOCH}_{2} \mathrm{CH}_{2} \mathrm{NH}-\right), \quad 4.60-4.80$ $(2 \mathrm{H}, \mathrm{br},-\mathrm{NHCOOCH}-), 4.98-5.12(2 \mathrm{H}, \mathrm{br},-\mathrm{NH}-), 5.88(2 \mathrm{H}, \mathrm{dd}, J=1.1$ and $\left.10.8 \mathrm{~Hz}, \mathrm{CH}_{2}=\mathrm{CH}-\right), 6.14\left(2 \mathrm{H}, \mathrm{dd}, J=10.6\right.$ and $\left.17.5 \mathrm{~Hz}, \mathrm{CH}_{2}=\mathrm{CH}-\right)$, $6.41\left(2 \mathrm{H}, \mathrm{dd}, J=1.1\right.$ and $\left.17.5 \mathrm{~Hz}, \mathrm{CH}_{2}=\mathrm{CH}-\right) .{ }^{13} \mathrm{C} \mathrm{NMR}(\delta$ in p.p.m.) $: 23.5$ $\left(-\mathrm{CH}_{2}\left(\mathrm{CH}_{2}\right)_{2} \mathrm{CH}_{2}-\right), 30.6\left(-\mathrm{CH}_{2}\left(\mathrm{CH}_{2}\right)_{2} \mathrm{CH}_{2}-\right), 39.9\left(-\mathrm{COOCH}_{2} \mathrm{CH}_{2} \mathrm{NH}-\right)$, $63.5\left(-\mathrm{COOCH}_{2} \mathrm{CH}_{2} \mathrm{NH}-\right), 74.3(-\mathrm{COOCH}-), 127.9\left(\mathrm{CH}_{2}=\mathrm{CH}-\right), 131.3$ $\left(\mathrm{CH}_{2}=\mathrm{CH}-\right), \quad 155.6 \quad(-\mathrm{CC}(=\mathrm{O}) \mathrm{NH}-), \quad 165.9 \quad\left(\mathrm{CH}_{2}=\mathrm{CHC}(=\mathrm{O})-\right)$. IR $\left(\mathrm{KBr}, \mathrm{cm}^{-1}\right): 1683(\mathrm{C}=\mathrm{O}$ in urethane), $1720(\mathrm{C}=\mathrm{O}$ in ester $), 3290$ (NH). EA: calcd C, 54.26; H, 6.58; N, 7.03; found C, 54.30; H, 6.62; N, 7.01.

\section{Polymerization of BACH (typical procedure)}

BACH $(99.6 \mathrm{mg}, 250 \mu \mathrm{mol})$ and AIBN $(410 \mu \mathrm{g}, 2.5 \mu \mathrm{mol})$ were added to a glass tube. After the tube was degassed and filled with nitrogen, 1,4-dioxane $(3.0 \mathrm{ml})$ was added. Then, the tube was cooled with liquid nitrogen, degassed and sealed.

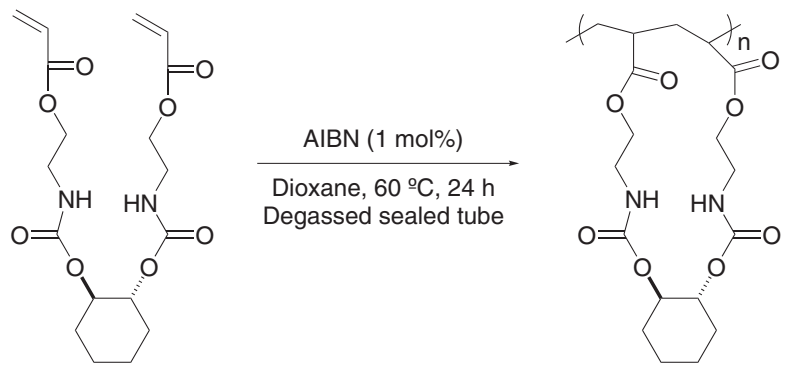

Scheme 1 Radical polymerization of BACH (trans-1,2-bisacryloyloxyethylcarbamoyloxycyclohexane).

Table 1 Radical polymerization of $\mathrm{BACH}$

\begin{tabular}{|c|c|c|c|c|c|c|}
\hline \multirow[b]{2}{*}{ Run } & \multirow[b]{2}{*}{ Conc. $\left(\mathrm{mol} \mathrm{l}^{-1}\right)$} & \multirow[b]{2}{*}{ Conv. $(\%)^{a}$} & \multicolumn{2}{|c|}{ Yield (\%) } & \multicolumn{2}{|c|}{$\mathrm{M}_{n}\left(\mathrm{M}_{w} \mathrm{M}_{n}\right)^{\mathrm{b}}$} \\
\hline & & & Soluble ${ }^{c}$ & Insoluble & $R I$ & RALLS \\
\hline 1 & 0.033 & 91 & 78 & 0 & 3100 (1.37) & $3700(1.22)$ \\
\hline 2 & 0.063 & 96 & 83 & 0 & 6400 (1.93) & $7800(2.28)$ \\
\hline 3 & 0.083 & 97 & 88 & 0 & $8200(2.27)$ & $8400(3.37)$ \\
\hline 4 & 0.125 & 97 & 85 & 0 & $14800(4.25)$ & 34700 (7.69) \\
\hline 5 & 0.400 & $>99$ & 0 & $>99$ & & \\
\hline
\end{tabular}

Abbreviations: BACH, trans-1,2-bisacryloyloxyethylcarbamoyloxycyclohexane; $M_{\mathrm{n}}$, number-average molecular weight; $M_{w}$, weight-average molecular weight; ${ }^{1} \mathrm{H}$ NMR, ${ }^{1} \mathrm{H}$ nuclear magnetic molecular weight; $M_{w}$, weight-average molecular weight; ${ }^{1} \mathrm{H}$ NMR, ${ }^{1} \mathrm{H}$
resonance; SEC, size-exclusion chromatography; THF, tetrahydrofuran.

resonance; SEC, size-exclusion chromatography; $\mathrm{THF}$, tetrahyd
aDetermined by ${ }^{1} \mathrm{H}$ NMR spectrometry $\left(400 \mathrm{MHz}^{\mathrm{C}} \mathrm{CDCl}_{3}\right)$.

bEstimated by SEC (THF, polystyrene standard).

cIsolated yield after precipitation with diethyl ether.

Isolated yield after Soxhlet extraction with THF.
The mixture was stirred at $60^{\circ} \mathrm{C}$ for $24 \mathrm{~h}$. The resulting polymer was isolated by precipitation with an excess amount of diethyl ether $(87.3 \mathrm{mg}, 87.6 \%)$.

${ }^{1} \mathrm{H} \quad \mathrm{NMR} \quad\left(\delta\right.$ in p.p.m.): $\quad 0.80-2.80 \quad\left(14 \mathrm{H}, \quad-\mathrm{CH}_{2} \mathrm{CH}(\mathrm{COOR})-\right.$, $\left.-\mathrm{CH}_{2}\left(\mathrm{CH}_{2}\right)_{2} \mathrm{CH}_{2}-\right), \quad 2.90-3.82$ (4H, br, $\left.-\mathrm{COOCH}_{2} \mathrm{CH}_{2} \mathrm{NH}-\right)$, 3.82-4.95 $\left(6 \mathrm{H},-\mathrm{COOCH}_{2} \mathrm{CH}_{2} \mathrm{NH}-,-\mathrm{NHCOOCH}-\right), 4.95-6.80$ (2H, br, $\left.-\mathrm{NH}-\right)$. ${ }^{13} \mathrm{C} \quad \mathrm{NMR} \quad(\delta$ in p.p.m. $): \quad 23.2-24.6 \quad\left(-\mathrm{CH}_{2}\left(\mathrm{CH}_{2}\right)_{2} \mathrm{CH}_{2}-\right), \quad 30.5-32.2$ $\left(-\mathrm{CH}_{2}\left(\mathrm{CH}_{2}\right)_{2} \mathrm{CH}_{2}-\right), \quad 32.1-38.5 \quad\left(-\mathrm{CH}_{2} \mathrm{CH}(\mathrm{COOR})-\right), \quad 38.5-44.0$ $\left(-\mathrm{CH}_{2} \mathrm{CH}(\mathrm{COOR})-,-\mathrm{COOCH}_{2} \mathrm{CH}_{2} \mathrm{NH}-\right), 62.0-66.8\left(-\mathrm{COOCH}_{2} \mathrm{CH}_{2} \mathrm{NH}-\right)$, 74.0-76.2 (-COOCH-), $\quad 155.8-157.6 \quad(-\mathrm{CC}(=\mathrm{O}) \mathrm{NH}-), \quad 174.0-176.8$ $\left(\mathrm{CH}_{2}=\mathrm{CHC}(=\mathrm{O})-\right) . \quad$ IR $\quad\left(\mathrm{KBr}, \quad \mathrm{cm}^{-1}\right): 1626 \quad(\mathrm{C}=\mathrm{O} \quad$ in urethane $)$, $1723(\mathrm{C}=\mathrm{O}$ in ester) and $3422(\mathrm{NH})$.

\section{RESULTS AND DISCUSSION}

\section{Radical polymerization of $\mathrm{BACH}$}

The diacrylate monomer $\mathrm{BACH}$ was prepared via the reaction of 2-acryloyloxyethylisocyanate and trans-1,2-cyclohexanediol in a manner similar to that of the analogous dimethacrylate monomer $\mathrm{BMCH}$. The ${ }^{1} \mathrm{H}$ NMR signal assigned to the $\mathrm{NH}$ group appeared at the identical chemical shift, ${ }^{17}$ suggesting that the conformation taken by $\mathrm{BACH}$ for ring closing is identical to that taken by $\mathrm{BMCH}$. The structure was validated by the NMR spectroscopic and elemental analyses.

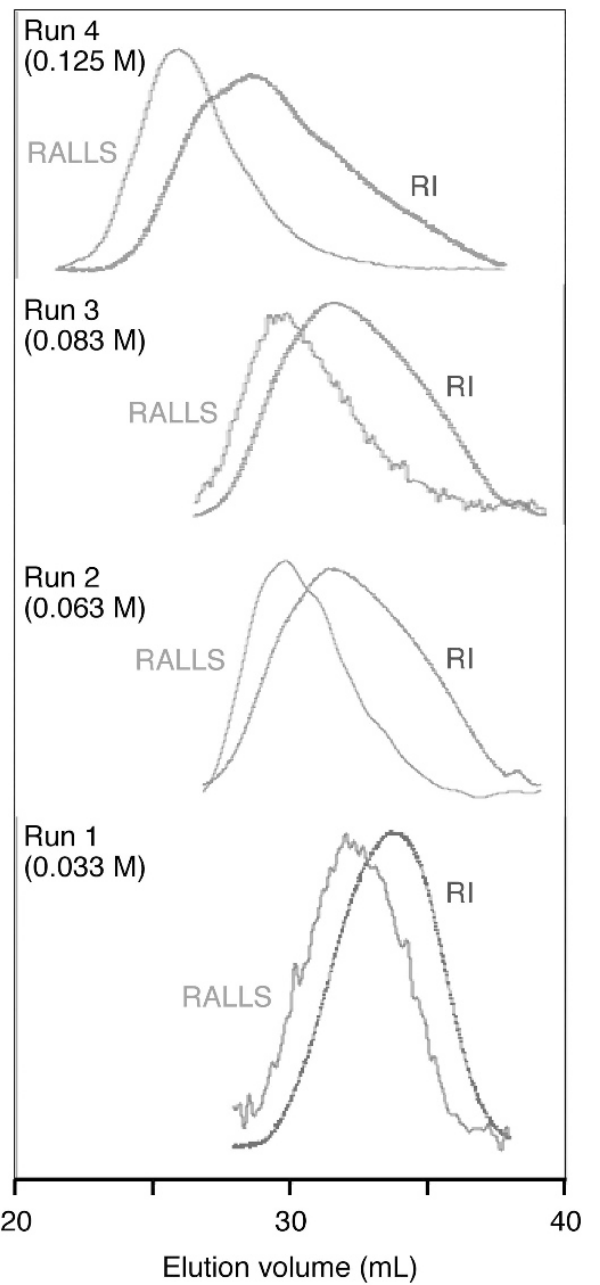

Figure 1 Refractive index (RI)- and right angle laser light scattering (RALLS)-detected size-exclusion chromatography (SEC) profiles of poly-BACH (trans-1,2-bisacryloyloxyethylcarbamoyloxycyclohexane) obtained under various concentrations $\left(2,2^{\prime}\right.$-azobisisobutyronitrile $(A I B N)=1 \mathrm{~mol} \%, 60^{\circ} \mathrm{C}$, $24 \mathrm{~h}$, degassed sealed tube). A full color version of this figure is available at Polymer Journal online. 
$\mathrm{BACH}$ was polymerized at $60^{\circ} \mathrm{C}$ in 1,4-dioxane under various concentrations using $\mathrm{AIBN}$ ( $1 \mathrm{~mol} \%$ to $\mathrm{BACH})$ as an initiator in degassed and sealed tubes (Scheme 1 and Table 1). Polymerization conducted under concentrations below $0.125 \mathrm{moll}^{-1}$ gave soluble products in high yields (runs 1-4), but polymerization conducted at $0.400 \mathrm{moll}^{-1}$ was insoluble in any common solvents (run 5). This tendency is almost identical to the polymerization of $\mathrm{BMCH}$, which yielded soluble and insoluble products at 0.082 and $0.200 \mathrm{moll}^{-1}$, respectively. ${ }^{17}$ The number-average molecular weight $\left(M_{\mathrm{n}}\right)$ of the polymers and the yields were lower compared with those of poly$\mathrm{BMCH}$ obtained under analogous conditions. The $M_{\mathrm{n}}$ values increased with the concentration, but the polydispersity indices $\left(M_{\mathrm{w}} / M_{\mathrm{n}}\right)$ also increased. This behavior implies that a higher concentration leads not only to smooth propagation but also undesired branching. The branching behavior was evaluated by size-exclusion chromatography profiles detected by refractive index and right angle laser light scattering (RALLS). The refractive index and RALLS profiles of the polymer obtained in run 1 have similar shapes, indicating that the polymer contains negligible branching (Figure 1). The shoulders of the refractive index profiles at the higher-molecular-weight region became clearer with the increase of the concentration, and the RALLS profiles shifted to significantly lower elution volume regions. This result supports that the highly selective cyclopolymerization proceeded under appropriately diluted conditions.

\section{Structure of poly-BACH}

The structure of poly-BACH was elucidated by NMR and MALDI-TOF mass spectroscopy. Figure 2 shows the ${ }^{1} \mathrm{H}$ and ${ }^{13} \mathrm{C}$ NMR spectra of poly-BACH obtained in run 1 in Table 1 . All signals are assignable to the plausible structure, and the spectra are almost analogous to those of poly-BMCH. ${ }^{17}$ Signals assignable to the acrylate moieties were not detected in either spectrum (i.e., the dd signals at 5.88-6.41 p.p.m. for ${ }^{1} \mathrm{H}$ NMR and signals at 128 and 131 p.p.m. for ${ }^{13} \mathrm{C}$ NMR). The clearly split signals of the methylene protons in both the formed ring ( $d$ and e) and the cyclohexane ring ( $i$ and $j$ ) suggest the constrained conformation by the ring structures. The efficient cyclization was confirmed by the ${ }^{1} \mathrm{H}$ NMR spectrum of the polymer obtained at low conversion. For example, the polymer obtained in an early stage $(0.083 \mathrm{M}, 30 \mathrm{~min}$, conversion $=53 \%)$ contains a negligible amount of the acrylate moieties (1.3\%) (Supplementary Figure S1). The ${ }^{1} \mathrm{H}$ NMR spectrum of the polymer obtained in $N, N$-dimethyl formamide contained trace signals of the acrylate moieties (1-2\%), suggesting the importance of the intramolecular hydrogen bonding of the urethane structure aligning the two acrylate moieties toward the same direction in a manner similar to that of $\mathrm{BMCH}$ (Supplementary Figure S2). ${ }^{17}$

The MALDI-TOF mass spectrum of poly-BACH obtained in run 1 in Table 1 is shown in Figure 3. Two series of the peaks with a difference of $398 \mathrm{Da}$, which corresponds with the molecular weight of $\mathrm{BACH}$, were observed. The intensity of the signals in the stronger series is $\sim 30$ times stronger than that of the signals in the weaker series. The simple spectrum indicates that most of the poly-BACH chains have the same initiating and terminating structures that originate from the selective cyclopolymerization. The peak with $m / z=3297.6 \mathrm{Da}$ is assignable to the octamer of $\mathrm{BACH}$ $(398.40 \times 8=3187.2)$. This spectrum was measured in the presence of $\mathrm{CF}_{3} \mathrm{COONa}$ as an ionization agent, and the peaks observed are ionized by $\mathrm{Na}^{+}$. The remaining mass, namely, $87.4 \mathrm{Da}$, does not match with the initiating and terminating structures originated from the radical from AIBN and the proton $(70 \mathrm{Da})$. This mass corresponds with the possible terminal structure originating from the solvent
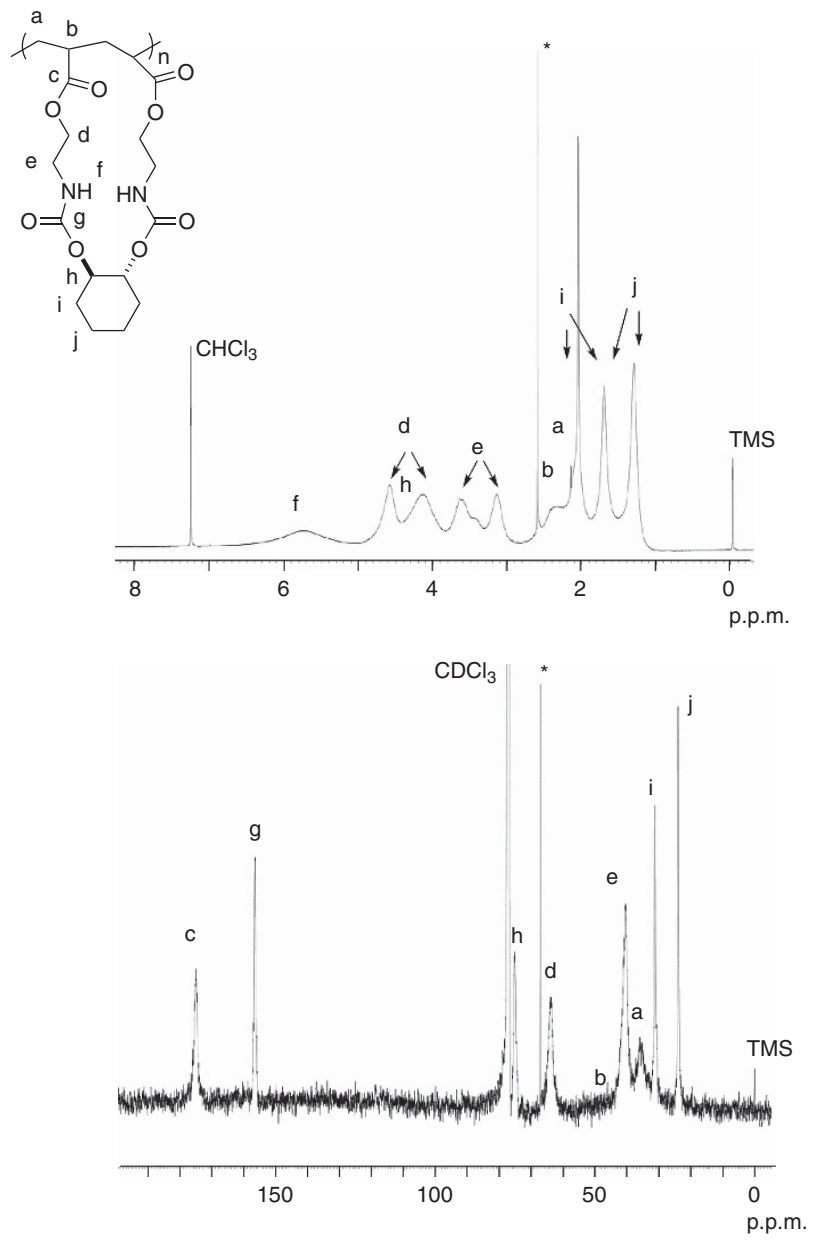

Figure $2{ }^{1} \mathrm{H}(400 \mathrm{MHz})$ and ${ }^{13} \mathrm{C}(100 \mathrm{MHz})$ nuclear magnetic resonance (NMR) spectra of poly-BACH (trans-1,2-bisacryloyloxyethylcarbamoyloxycyclohexane) obtained in run 1 in Table $1\left(\mathrm{CDCl}_{3}\right.$, room temperature (rt)). Asterisks indicate the signal of the remaining 1,4-dioxane.

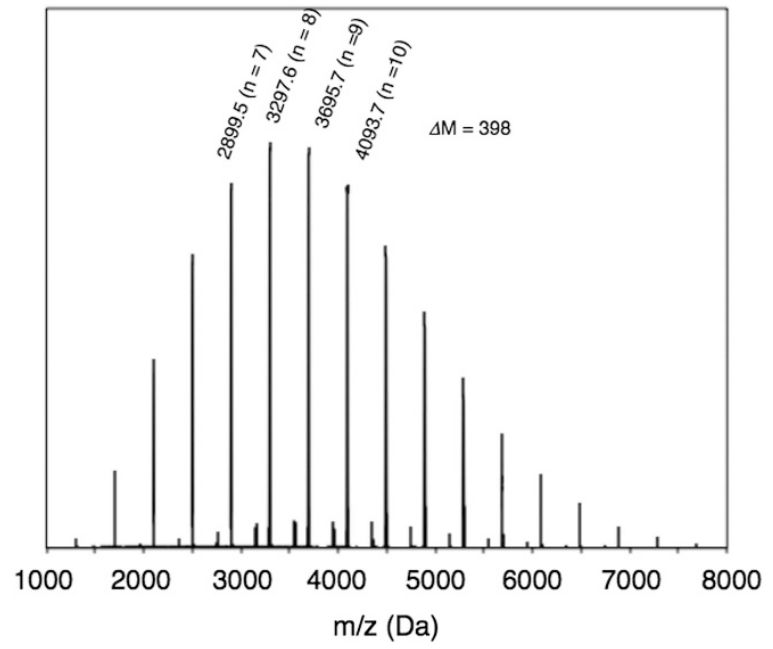

Figure 3 Matrix-assisted laser desorption/ionization time-of-flight (MALDI-TOF) mass spectrum of poly-BACH (trans-1,2-bisacryloyloxyethylcarbamoyloxycyclohexane) obtained in run 1 in Table 1. 
1,4-dioxane $(88.1 \mathrm{Da})$ and suggests the initiation from the radical produced via hydrogen abstraction from 1,4-dioxane. The MALDI-TOF mass spectrum of the polymer obtained in $N, N$-dimethyl formamide also shows the efficient cyclization and the initiation from $N, N$-dimethyl formamide (Supplementary Figure S3). This MALDI-TOF mass spectroscopic analysis supports the welldefined structure produced via highly selective cyclopolymerization. If the polymerization was accompanied by intermolecular branching, the resulting chain would have multiple initiating and terminating groups that result in another series of the peaks. The minor series of the signals implies a trace degree of an unexpected reaction, whereas we could not identify the structure. The masses do not match with the polymers bearing two initiating and terminating structures.

\section{CONCLUSIONS}

Radical polymerization of a bisacrylate monomer $\mathrm{BACH}$ proceeded via selective cyclopolymerization to give 19-membered rings under appropriate conditions. The high yield and the selective cyclopolymerization, which is typically inconsistent, may be achieved by designing the monomer structure to align the two acrylate moieties to a conformation suitable to cyclopolymerization. The facile one-step method for preparation of the monomer is also an advantage of this cyclopolymerization. We are now searching for applications of this polymer depending on the characteristic large ring structure.

\section{CONFLICT OF INTEREST}

The authors declare no conflict of interest.

\section{ACKNOWLEDGEMENTS}

We thank Mr Takeyoshi Takahashi (Yamagata University) for his contribution to the elemental analysis. We also thank Dr Daisuke Takeuchi and Mr Masato Koizumi (Tokyo Institute of Technology) for their kind contribution to the MALDI-TOF MS analysis.

1 Butler, G. B. Cyclopolymerization. J. Polym. Sci. Part A 38, 3451-3461 (2000)

2 Kodaira, T. Structural control during the cyclopolymerization of unconjugated dienes. Prog. Polym. Sci. 25, 627-676 (2000).

3 Takeuchi, D. Stereo-controlled synthesis of polyolefins with cycloalkane groups by using late transition metals. Polym. J. 44, 919-928 (2012).

4 Tunca, U. \& Yagci, Y. Crown ether-containing polymers. Prog. Polym. Sci. 19, 233-286 (1994).
5 Izatt, R. M., Pawlak, K., Bradshaw, J. S. \& Bruening, R. L. Thermodynamic data for macrocycle interaction with cations and anions. Chem. Rev. 91, 1721-2085 (1991).

6 Gokel, G. W., Leevy, W. M. \& Weber, M. E. Crown ethers: sensors for ions and molecular scaffolds for materials and biological models. Chem. Rev. 104, 2723-2750 (2004).

7 Szejtli, J. Introduction and general overview of cyclodextrin chemistry. Chem. Rev. 98, 1743-1753 (1998).

8 Uekama, K., Hirayama, F. \& Irie, T. Cyclodextrin drug carrier systems. Chem. Rev. 98, 2045-2076 (1998).

9 Yokota, K., Matsumura, M., Yamaguchi, K. \& Takada, Y. Synthesis of polymers with benzo-19-crown-6 units via cyclo-polymerization of divinyl ethers. Makromol. Chem. Rapid Commun. 4, 721-724 (1983).

10 Sakai, R., Satoh, T., Kakuchi, R., Kaga, H. \& Kakuchi, T. Helicity induction of polyisocyanate with a crown cavity on the main chain synthesized by cyclopolymerization of $\alpha, \omega$-diisocyanate. Macromolecules 37, 3996-4003 (2004).

11 Kakuchi, T., Haba, O. \& Yokota, K. Cyclopolymerization of divinyl ethers. Synthesis and the cation-binding property of poly(crown ether)s. Macromolecules 25, 4854-4858 (1992).

12 Rahman, M. S., Hashimoto, T. \& Kodaira, T. Cationic cyclopolymerization of new divinyl ethers: the effect of ether and ester neighboring functional groups on their cyclopolymerization tendency. J. Polym. Sci. Part A 41, 281-292 (2003).

13 Hashimoto, T., Watanabe, K. \& Kodaira, T. Cationic cyclopolymerization of 1,2-bis(2-vinyloxyethoxy) benzenes: Introduction of bulky substituents to increase cyclopolymerization tendency. J. Polym. Sci. Part A 42, 3373-3379 (2004).

14 Sanda, F., Kawano, T. \& Masuda, T. Cyclopolymerization of amino acid-based diynes and properties of the obtained polymers. Chiral recognition and metal ion extraction. Polym. Bull. 55, 341-347 (2005).

15 Nagai, A., Ochiai, B. \& Endo, T. Cyclopolymerization of bisacrylamide derived from $\alpha$-pinene through larger chiral ring formation. Maromolecules 38, 2547-2549 (2005).

16 Costa, A. I., Barata, P. D. \& Prata, J. V. Radical cyclopolymerization of a divinylbenzyl-p-tert-butylcalix[4]arene derivative. React. Funct. Polym. 66, 465-470 (2006)

17 Ochiai, B., Ootani, Y. \& Endo, T. Controlled cyclopolymerization through quantitative 19-membered ring formation. J. Am. Chem. Soc. 130, 10832-10833 (2008).

18 Edizer, S., Veronesi, B., Karahan, O., Aviyente, V., Degirmenci, I., Galbiati, A. \& Pasini, D. Efficient free-radical cyclopolymerization of oriented styrenic difunctional monomers. Macromolecules 42, 1860-1866 (2009).

19 Sharma, A. K., Cornaggia, C. \& Pasini, D. Controlled RAFT cyclopolymerization of oriented styrenic difunctional monomers. Macromol. Chem. Phys. 211, 2254-2259 (2010).

20 Ochiai, B., Ito, S. \& Endo, T. Chiral interaction between aromatic aldehydes and a polymer bearing large chiral rings obtained via cyclopolymerization of bisacrylamide. Polym. J. 42, 138-141 (2010).

$21 \mathrm{Li}, \mathrm{C}$., Liu, H. \& Li, Y. 1,2,3-Triazole-incorporated diacrylate monomer for free radical cyclopolymerization through large ring formation. Macromol. Chem. Phys. 212, 1050-1055 (2011).

22 Elliott, B. J., Scranton, A. B., Cameron, J. H. \& Bowman, C. N. Characterization and polymerization of metal complexes of poly(ethylene glycol) diacrylates and the synthesis of polymeric pseodocrown ethers. Chem. Mater. 12, 633-642 (2000).

23 Terashima, T., Kawabe, M., Miyabara, Y., Yoda, H. \& Sawamoto, M. Polymeric pseudo-crown ether for cationic recognition via cation template-assisted cyclopolymerization. Nat. Commun. 4, 2321 (2013).

24 Jia, Y., Liu, L., Lei, B., Li, J. \& Zhu, X. X. Crown ether cavity-containing copolymers via controlled alternating cyclopolymerization. Macromolecules 44, 6311-6317 (2011).

25 Zou, L., Liu, J., Zhang, K., Chen, Y. \& Xi, F. Cyclopolymerization of $\alpha, \omega$-heterofunctional monomers containing styrene and maleimide moieties. J. Polym. Sci. Part A 52 , 330-338 (2014)

Supplementary Information accompanies the paper on Polymer Journal website (http://www.nature.com/pj) 